\title{
Distinct Mechanisms for Size Tuning in Primate Visual Cortex
}

\author{
Farran Briggs ${ }^{1,2}$ and W. Martin Usrey ${ }^{1}$ \\ ${ }^{1}$ Center for Neuroscience, University of California, Davis, Davis, California 95618, and ${ }^{2}$ Department of Physiology and Neurobiology, Dartmouth Medical \\ School, Lebanon, New Hampshire 03756
}

Most neurons in primary visual cortex (V1) are selective for stimulus size, a property with important implications for salient feature detection. Size selectivity involves dynamic interactions between neuronal circuits that establish the classical (center) and extraclassical (surround) of a neuron's receptive field. Although much is known about the tuning properties and stimulus selectivity of the center and surround subunits, relatively little is known about how these subunits interact to achieve size selectivity. To address this question, we examined the temporal dynamics of size selectivity in two classes of pyramidal neurons at similar hierarchical processing stages in V1 of alert monkeys. These two classes were comprised of neurons in cortical layer 6 with identified projections to the lateral geniculate nucleus. While both neuronal groups displayed comparable levels of size selectivity, the temporal dynamics of their tuning differed significantly. We compared the size tuning profiles of each cell type with a series of sum-of-Gaussian models and discovered that the receptive fields of neurons with fast-conducting axons contained an excitatory center and a suppressive surround with similar onset timing. In contrast, neurons with slow-conducting axons used two center components - an early wide-field component and a delayed narrow-field component that increased activity-in addition to the surround component. The early, wide-field component represents a novel mechanism for cortical neurons to integrate contextual information. These results demonstrate that size tuning in cortical neurons is established via multiple unique mechanisms, dictated by the rich circuit architecture in which neurons are embedded.

\section{Introduction}

Neurons in primary visual cortex (V1) are defined principally by their physiological responses to visual stimulation of their classical receptive fields. For many neurons, stimuli that extend beyond the classical receptive field and into the extraclassical surround suppress spiking activity (Knierim and Van Essen, 1992). Tuning for stimulus size, or size selectivity, emerges via interactions between the neuronal circuits that establish the receptive field center and surround. Given the behavioral utility of size selectivity for detecting salient features in the visual environment (Mareschal and Shapley, 2004), it is important to understand the mechanisms that underlie size selectivity.

A rich architecture of neuronal circuits-including feedforward thalamic input, local excitatory and inhibitory input, and feedback input from extrastriate cortical areas - underlie the response properties of V1 neurons (Felleman and Van Essen, 1991; Callaway, 2004; Sincich and Horton, 2005). Consequently, multiple circuits likely contribute to center/surround interactions in

Received May 5, 2011; revised July 18, 2011; accepted July 19, 2011.

Author contributions: F.B. and W.M.U. designed research; F.B. performed research; F.B. analyzed data; F.B. and W.M.U. wrote the paper.

This work was supported by NIH Grants EY013588, EY018683, and EY012576. We thank Mark Goldman for consultation on the model and Katie Neverkovec and Dan Sperka for technical assistance.

Correspondence should be addressed to W. Martin Usrey, Center for Neuroscience, University of California, Davis, 1544 Newton Court, Davis, CA 95618. E-mail: wmusrey@ucdavis.edu.

DOI:10.1523/JNEUROSCI.2268-11.2011

Copyright $\odot 2011$ the authors $\quad 0270-6474 / 11 / 3112644-06 \$ 15.00 / 0$
V1 (Angelucci et al., 2002; Carandini et al., 2002; Cavanaugh et al., 2002a; Levitt and Lund, 2002; Bair et al., 2003; Ozeki et al., 2004; Rust et al., 2005; Angelucci and Bressloff, 2006; Sadakane et al., 2006; Sullivan and de Sa, 2006; Chen et al., 2007; Durand et al., 2007; Tailby et al., 2007; Liu et al., 2011). Given the complexity of V1 network connectivity, size tuning among different neuronal populations could rely on distinct mechanisms. Support for this idea includes the observation that size selectivity varies across V1 laminae (Sceniak et al., 1999, 2001; Jones et al., 2001; Levitt and Lund, 2002).

To provide a quantitative understanding of the mechanisms underlying center and surround contributions to size selectivity, we compared the temporal properties of center and surround interactions in two physiologically distinct populations of corticogeniculate neurons in V1 of alert macaque monkeys (Briggs and Usrey, 2009). These neurons were selected because they occupy similar positions in the V1 laminar hierarchy-they are all located in cortical layer 6, provide feedback signals to the LGN, and receive local excitatory input from layer 4C (Lund et al., 1975; Fitzpatrick et al., 1994; Briggs and Callaway, 2001; Briggs and Usrey, 2007, 2009). Despite these similarities, our results reveal marked differences between the cell groups in their spatial and temporal interactions for achieving size selectivity.

\section{Materials and Methods}

All procedures conformed to NIH guidelines and were approved by the Institutional Animal Care and Use Committee at UC Davis. Single-unit 
recordings were made from identified corticogeniculate neurons in two alert male macaque monkeys. Data from these animals also contributed to a study examining the visual physiology of corticogeniculate neurons (Briggs and Usrey, 2009). This previous study did not examine the temporal dynamics of size selectivity.

Corticogeniculate neurons were identified by antidromic activation following electrical stimulation to the LGN and a collision test (Briggs and Usrey, 2007, 2009). Visual stimuli were centered over the receptive fields of recorded neurons while animals maintained fixation. Stimulus presentation began $200 \mathrm{~ms}$ following fixation onset and continued for $1.4 \mathrm{~s}$, followed by $1.4 \mathrm{~s}$ of mean gray. Eye position was continuously monitored; trials were aborted if the eye deviated by $>0.35^{\circ}$. Stimuli were drifting sinusoidal gratings ( $70 \%$ contrast, $4 \mathrm{~Hz}$, preferred spatial frequency, and preferred orientation). Stimuli varied in size from $0.2^{\circ}$ to $10^{\circ}$ in 12 steps. Each stimulus sequence was presented a minimum of three times.

Neurons were classified as simple or complex based on their $\mathrm{fl}: \mathrm{f0}$ response to optimal drifting gratings. Area-summation tuning curves were fit with a difference-of-Gaussians equation (Sceniak et al., 1999),

$$
\begin{array}{r}
R(S)=R_{0}+K_{c} \int_{-S / 2}^{S / 2} \exp -(2 y / a)^{2} \times d y-K_{s} \int_{-S / 2}^{S / 2} \exp \\
-(2 y / b)^{2} \times d y,
\end{array}
$$

where $S$ represents stimulus size, $R_{0}$ represents spontaneous firing rate, $K_{\mathrm{c}}$ and $K_{\mathrm{s}}$ are amplitudes of center and surround Gaussians, respectively, and $a$ and $b$ represent $\sigma$ values for the excitatory center and suppressive surround Gaussians.

The strength of surround suppression was calculated using a suppression index,

SI $=1-$ (response to largest-size stimulus/

response to preferred-size stimulus).

The temporal dynamics of size tuning were studied using a subset of corticogeniculate complex cells with suppression index values $>0.2$. Simple cells were excluded due to modest levels of suppression (mean $\mathrm{SI}=0.28 \pm 0.05 \mathrm{SEM}$ ). The remaining sample contained two groups of complex cells, those with short antidromic-activation latencies $(<8 \mathrm{~ms}$; Fast cells) and those with longer latencies ( $>15 \mathrm{~ms}$; Slow cells). For each neuron, PSTHs showing the time course of visual responses were calculated for preferred-size gratings and large gratings $\left(10^{\circ}\right)$ and normalized to the peak response. To compare PSTHs across neurons, individual histograms were shifted by the neuron's latency to reach half-maximum response to the preferred-size grating. Histograms were averaged for each group and smoothed.

A modified energy model (Adelson and Bergen, 1985) was constructed to examine the temporal dynamics of size tuning. We used a sum-of-Gaussians model rather than a divisive- or gain-normalization model (Sceniak et al., 1999, 2001; Cavanaugh et al., 2002a; Bonin et al., 2005; Webb et al., 2005), because the sum-of-Gaussians model allowed for a more straightforward incorporation of additional, separable receptive-field components. The model consisted of two phase-shifted spatial filters generated by summing two or three Gaussians, each defined by a unique temporal filter. The response of the model was computed by taking the dot product of each spatial filter and the stimulus $(4 \mathrm{~Hz}$ drifting sinusoidal grating, preferred spatial frequency) and convolving the resulting output with the temporal filters. The two resultant outputs were then squared and summed to generate the model cell's response as a spike rate. Model responses were generated for two stimuli, a preferred-size grating $\left(\sigma=1^{\circ}\right)$ and a large-size grating $\left(\sigma=5^{\circ}\right)$.

Two versions of the model were constructed: a 2-D model in which the spatial filters each consisted of a sum of two Gaussians representing the receptive field's excitatory-center and suppressive-surround components; and a 3-D model that incorporated an additional Gaussian representing a wide-field excitatory component. Each Gaussian component of the spatial filters was defined by a fixed width: excitatory center $\sigma=1^{\circ}$, suppressive surround $\sigma=5^{\circ}$, wide-field excit- atory center $\sigma=5^{\circ}$. These values approximated the corresponding $\sigma$ values from the fits of the experimental size tuning curves (Fast cells: excitatory center $\sigma=0.64^{\circ}$, suppressive surround $\sigma=4.26^{\circ}$; Slow cells: excitatory center $\sigma=0.57^{\circ}$, suppressive surround $\sigma=5.28^{\circ}$ ). The amplitudes of each Gaussian were free parameters constrained by biologically realistic limits.

The temporal filters for each component of the spatial filters were formulated after DeAngelis et al. (1995),

$$
f(t)=\alpha^{2} \exp (-\alpha \times t)-\beta^{2} \exp (-\beta \times t),
$$

where $\alpha$ and $\beta$ define the rise and reversal times of each temporal filter. These parameters were allowed to vary within biologically realistic constraints.

A least-squares minimization protocol (fminsearch, Matlab) was used to optimize and assign values to each of the free parameters by comparing the output of the 2-D and 3-D models with the averaged and smoothed PSTHs for each neuronal class.

\section{Results}

Single-unit recordings were made from 35 identified corticogeniculate neurons in V1 of two alert macaque monkeys performing a fixation task while drifting sinusoidal gratings (70\% contrast, $4 \mathrm{~Hz}$, preferred orientation and spatial frequency) varying in size were presented centered over the neuron's receptive field. Nine neurons were classified as simple cells and 26 were classified as complex cells based on their $\mathrm{f1}$ :f0 response to optimal drifting gratings. All simple cells and two complex cells were excluded from further analyses because they did not exhibit sufficient surround suppression, defined by suppression index values $>0.2$ (Materials and Methods).

An examination of the antidromic-activation latency and visual-response latency for complex corticogeniculate neurons revealed two distinct groups: neurons with fast conducting axons and short visual response latencies (Fast cells) and neurons with slower conducting axons and longer visual response latencies (Slow cells; Fig. 1 A) (Briggs and Usrey, 2009). Area-summation tuning curves for both groups of cells appeared similar in overall shape (Fig. 1C,D). Namely, responses initially increased as stimulus size increased and then decreased to larger stimuli (preferred size: Fast cells $=0.64^{\circ} \pm 0.11^{\circ}$, SEM; Slow cells $=0.57^{\circ} \pm 0.11^{\circ}, \mathrm{SEM} ; p=0.7$, rank-sum test). The strength of surround suppression was also similar for the two groups, as quantified with a suppression index (SI: Fast cells $=0.43 \pm 0.05$, SEM; Slow cells $=0.51 \pm 0.07$, SEM; $p=0.4$, rank-sum test).

Differences between Fast and Slow cells were immediately apparent upon examination of the temporal dynamics of size tuning. As expected, latencies to the half-maximal response for Fast cells were shorter for both preferred-size and largesize gratings compared to Slow cells $\left(p=1.1 \times 10^{-5}\right.$, KruskalWallis test). More importantly, Fast and Slow cells differed from each other in terms of the relative timing of their responses to preferred-size and large-size gratings. Specifically, Fast cells responded more quickly to preferred-size gratings than to large-size gratings, while Slow cells responded more quickly to large-size gratings than to preferred-size gratings (Fig. $1 B$ ). These effects were evident in individual and population PSTHs (Fig. 2A-D). Accordingly, both Fast and Slow cell response-latency distributions (preferred-size latency large-size latency) were shifted significantly from $0(p<0.01$, Kolmogorov-Smirnov test) with Fast cells responding $\sim 4 \mathrm{~ms}$ faster to the preferred-size stimulus and Slow cells responding $\sim 5 \mathrm{~ms}$ faster to the large-size stimulus. An examination of the cumulative difference in responses to preferred-size and largesize gratings over time further revealed the striking difference 

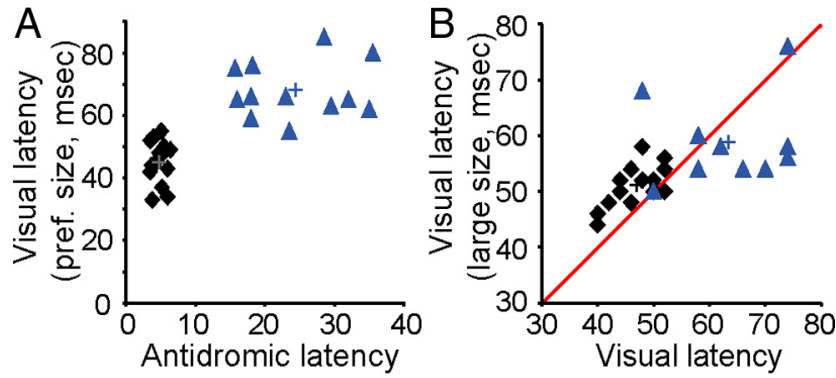

(msec)
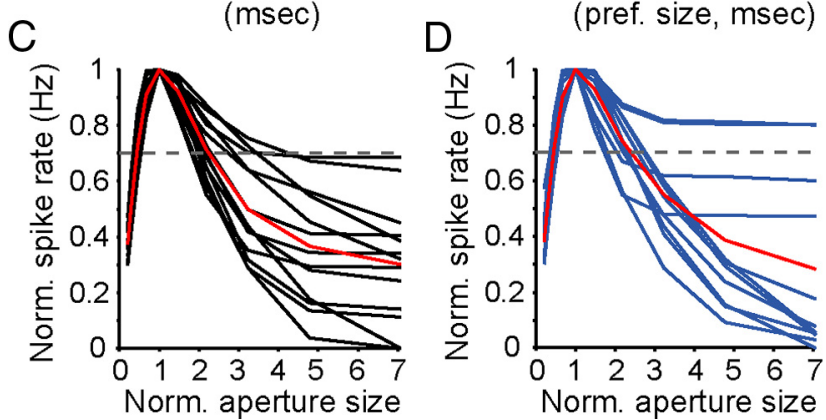

Figure 1. Latencies and area-summation tuning curves for two populations of corticogeniculate neurons. $A$, Antidromic latency versus latency to half-maximum response to preferred-size grating for 26 corticogeniculate neurons. Black diamonds, Fast cells $(n=14)$; blue triangles, Slow cells $(n=12)$. Crosses indicate mean latencies (Fast cells: mean antidromic latency $=4.75 \pm 0.25 \mathrm{~ms}$, SEM; mean visual latency $=45.07 \pm 1.71 \mathrm{~ms}$, SEM; Slow cells: mean antidromic latency $=24.41 \pm 2.14 \mathrm{~ms}$, SEM; mean visual latency $=68.08+/ 2.59 \mathrm{~ms}$, SEM). $\boldsymbol{B}$, Latency to half-maximum response to preferred-size versus large-size gratings for 14 Fast cells (mean preferred-size latency $=46.7 \pm 1.2 \mathrm{~ms}$, SEM; mean large-size latency $=$ $51.0 \pm 1.1 \mathrm{~ms}, \mathrm{SEM}$ ) and 10 Slow cells (mean preferred-size latency $=63.4 \pm 3.3 \mathrm{~ms}, \mathrm{SEM}$; mean large-size latency $=58.8 \pm 2.6 \mathrm{~ms}$, SEM; conventions as in $A$ ). Red line represents unity. C, Area-summation tuning curves for 14 Fast cells where stimulus size was normalized to the preferred-size grating and reported as size relative to preferred. Red curve, Sample mean; dashed line, cutoff for inclusion in further analysis based on suppression index. $\boldsymbol{D}$, Areasummation tuning curves for 12 slow cells, conventions as in $\boldsymbol{C}$.

in size-tuning dynamics between Fast and Slow cells. Slow cells exhibited an early and transient increase in activity to large stimuli before suppression that was not evident among Fast cells (Fig. 2E).

To explore the composition of receptive-field subunits giving rise to size tuning in Fast and Slow cells, we built a modified energy model with spatial filters defined by a sum-of-Gaussians representing the excitatory center and suppressive surround receptive-field components, each with independent temporal dynamics defined by separate temporal filters. A 2-D model fit the Fast-cell data quite well (Fig. 3A, left). The optimal temporal filters revealed nearly coincident latencies for the onset of the excitatory center and suppressive surround components of the receptive field (Fig. $3 A$, right bottom panel). When the $2-\mathrm{D}$ model was fit to the Slow-cell data, it failed to capture the delay in suppression (Fig. 3B). With similarly timed excitatorycenter and suppressive-surround kinetics (Fig. 3B, right bottom panel), the 2-D model failed to reproduce a sufficient shift in the response latency between preferred-size and large-size gratings (difference in timing to half-maximum response $=$ $-1 \mathrm{~ms}$; Fig. 3B, left).

In contrast, a 3-D model, which incorporates an additional wide-field excitatory center Gaussian, fit the Slow-cell data quite well (Fig. 3C). The wide-field, excitatory-center component displayed faster and more transient kinetics compared to the narrow-field, excitatory-center and suppressive-surround components (Fig. 3C, right bottom panel). The 3-D model correctly
A
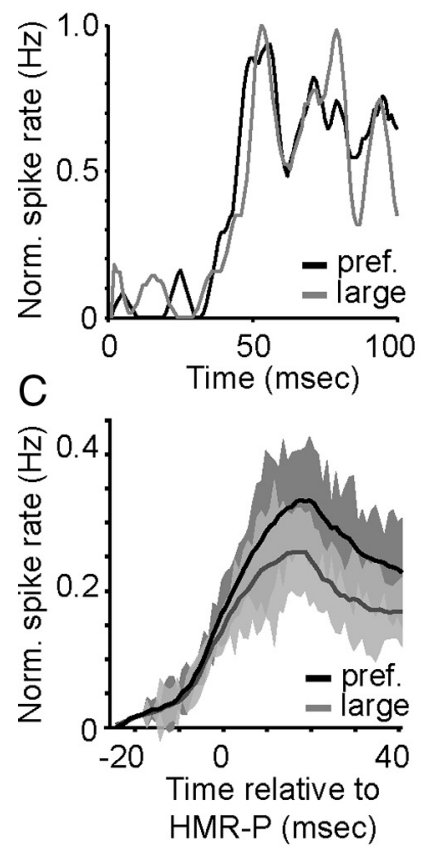

B

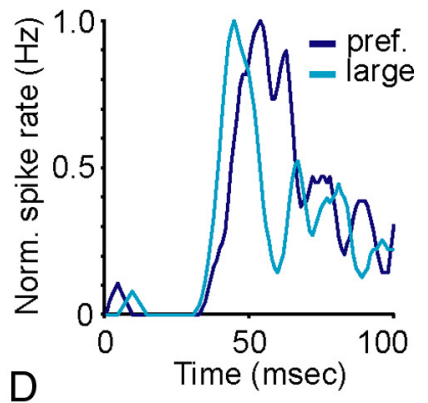

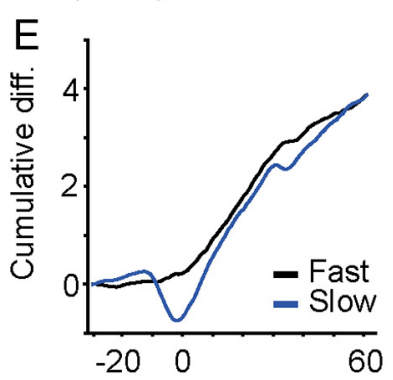

Time relative to HMR-P (msec)

Figure 2. Temporal dynamics of size tuning for Fast and Slow cells. $\boldsymbol{A}, \boldsymbol{B}$, PSTHs for individual Fast cells $(\boldsymbol{A})$ and Slow cells $(\boldsymbol{B})$ in response to preferred-size (black, dark blue) and large-size (gray, light blue) gratings. C, D, Mean PSTHs for responses to preferred-size (black, dark blue) and large-size (gray, light blue) gratings for 14 Fast cells $(\boldsymbol{C})$ and 10 Slow cells $(\boldsymbol{D})$. Zero represents latency to half-maximum response for preferred-size grating (HMR-P). Dark and light shaded regions represent SEMs. $\boldsymbol{E}$, Cumulative differences in preferred- and large-size grating responses for Fast cells (black) and Slow cells (blue) over time relative to HMR-Ps for each population.

captured the difference in response latency between the largesize and preferred-size gratings (difference in timing to halfmaximum response $=+5 \mathrm{~ms}$; Fig. $3 C$, left). To compare the response latencies computed by each model for Fast and Slow cells, we examined the cumulative difference in model responses to preferred-size and large-size gratings over time. Figure $3 D$ shows the cumulative latency differences for the 2-D model description of Fast cells, the 2-D model description of Slow cells, and the 3-D model description of Slow cells. A comparison of Figures $3 D$ and $2 E$ illustrates that the 2-D and 3-D models each capture the temporal dynamics of size tuning in Fast and Slow cells, respectively.

\section{Discussion}

This study examined the temporal dynamics of size tuning in similar, but distinct, populations of $\mathrm{V} 1$ corticogeniculate neurons to provide a quantitative understanding of the range of mechanisms underlying center/surround interactions for size selectivity. By combining empirical and modeling approaches, we 

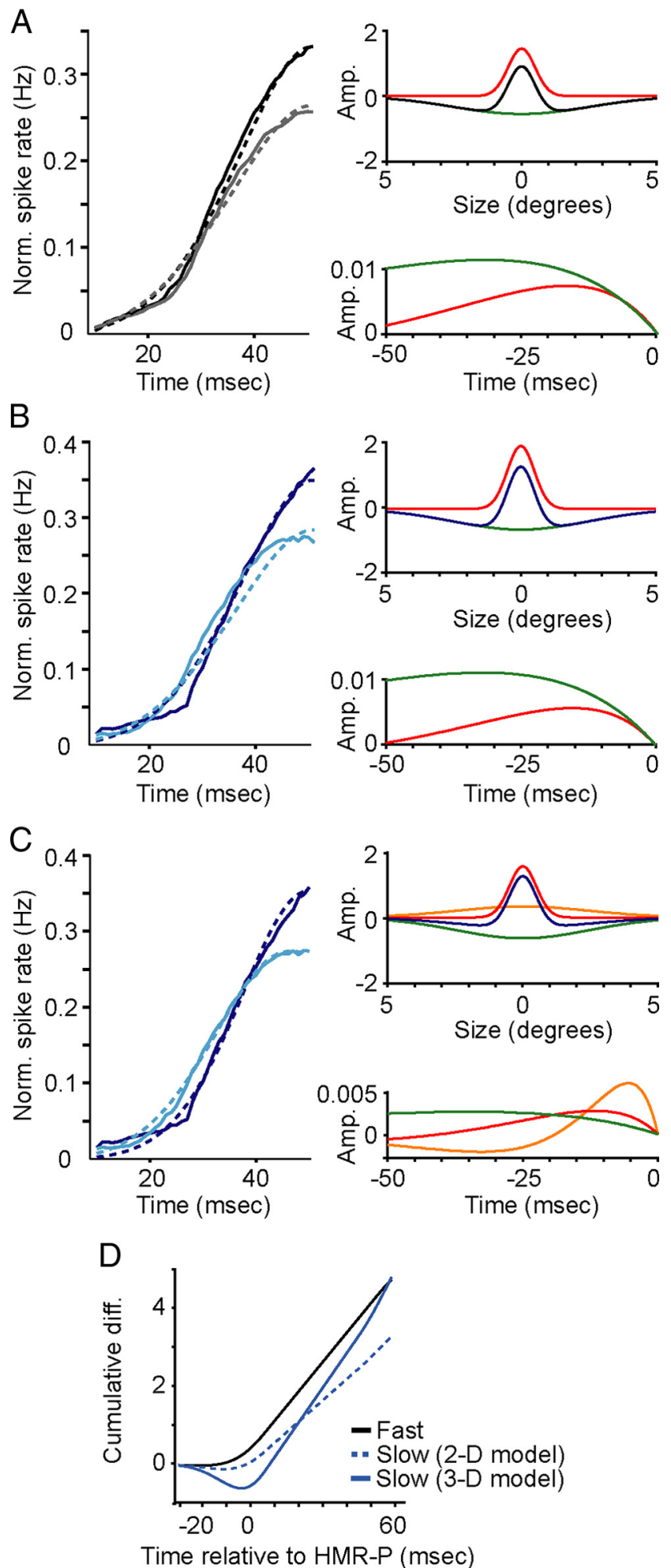

Figure 3. Model fits to Fast- and Slow-cell PSTHs. $A$, Left panel illustrates population mean PSTHs for Fast-cell responses to preferred-size (solid black) and large-size (solid gray) gratings compared to model predictions for preferred-size (dashed black) and large-size (dashed gray) grating responses. Timescale optimized to illustrate early response dynamics ( $10-50 \mathrm{~ms}$ after stimulus onset). Right, spatial (top) and temporal (bottom) filters: excitatory center in red, suppressive surround in green, sum in black (top) for the 2-D model. B, Left, Population mean PSTHs for Slow-cell responses to preferred-size (dark blue) and large-size (light blue) gratings compared to the 2-D model predictions for preferred-size (dashed dark blue) and large-size (dashed light blue) grating responses. Right, Spatial (top) and temporal (bottom) filters with same conventions as $A$ except the sum of the two spatial filters is depicted in dark blue. $C$, Left, Population PSTHs for Slow cells with the 3-D model predictions (conventions as in $\boldsymbol{B}$ ). Right, demonstrate that the number of subunits contributing to center/ surround interactions and the timing of excitation and suppression differs between Fast and Slow cell types. We identified two types of center/surround interactions: one involving near simultaneous center and surround activation (Fast cells), and the other involving fast and transient wide-field center activation followed by lagged suppression and focal excitation (Slow cells). We constructed two models describing the center and surround components of Fast and Slow cell receptive fields and simulation results indicate that Fast and Slow cells develop size selectivity via different mechanisms, as Fast cells are best fit by a model with center excitation and surround suppression sharing similar temporal dynamics, while Slow cells are best fit by a model with fast and transient wide-field center activation followed by similarly timed center excitation and surround suppression. These results indicate that distinct mechanisms underlie size tuning in V1, likely involving different combinations of subcortical, local circuit, and extrastriate inputs.

Stimulus contrast can influence both the spatial and temporal properties of center/surround interactions in V1 (Kapadia et al., 1999; Sceniak et al., 1999; Cavanaugh et al., 2002b; Nolt et al., 2004; Webb et al., 2005; Sadakane et al., 2006; Sceniak et al., 2006; Ichida et al., 2007; Tailby et al., 2007; Cai et al., 2008; Wang et al., 2009; Schwabe et al., 2010). We therefore recorded neuronal responses at a constant contrast (70\%) to ensure that any dynamics observed in the data were not attributable to contrast-mediated changes in response timing. Similarly, the relative strength and timing of center/surround interactions can vary depending on the orientation of stimuli in the center and surround regions of receptive fields (Valberg et al., 1985; Nothdurft et al., 1999; Jones et al., 2001, 2002; Angelucci et al., 2002; Cavanaugh et al., 2002b; Bair et al., 2003; Webb et al., 2003, 2005; Smith et al., 2006; Naito et al., 2007; Tailby et al., 2007). We therefore used a single grating that varied in size to ensure that any differences in the temporal dynamics of size tuning cannot be attributed to nonhomogenous pattern effects.

The data and model predictions provide important insight into the neuronal circuits mediating center/surround interactions. First, because the center and surrounds of Fast cells display similar kinetics, they could be jointly inherited from a common source. One potential source is direct, geniculocortical input. Along these lines, recent work indicates that Fast cells, but not Slow cells, receive direct suprathreshold geniculate input (Briggs and Usrey, 2007). Moreover, LGN neurons in the primate, particularly magnocellular neurons, display comparable levels of extraclassical suppression (Solomon et al., 2006; Alitto and Usrey, 2008; Camp et al., 2009) and the relative timing of center excitation and surround suppression in magnocellular neurons is similar to that of Fast cells (Alitto and Usrey, 2008). Thus, direct geniculate input has many of the properties needed to establish the center and surround receptive-field components of Fast cells. Second, the distinct temporal profiles of suppression in Fast and Slow cells-one coincident with center excitation, the other lagged relative to center excitation-suggest cell-type specific circuits for suppression. Third, the differing numbers of excitatory subunits for Fast and Slow cells (i.e., a single subunit for

$\leftarrow$

Spatial (top) and temporal (bottom) filters as in $\boldsymbol{B}$ with added wide-field excitatory center component in orange. $\boldsymbol{D}$, Cumulative differences in preferred- and large-size grating responses for the 2-D model Fast cell (black), the 2-D model Slow cell (dashed blue), and the 3-D model Slow cell (solid blue). 
Fast cells, vs an early transient wide-field subunit that precedes a narrow-field subunit for Slow cells) indicate there are distinct circuits for center excitation in these cell types. Possible sources contributing to wide-field excitation include neighboring layer 6 neurons with linear spatial summation (Gilbert, 1977; Grieve and Sillito, 1995; Sceniak et al., 2001; Levitt and Lund, 2002). Along these lines, past studies reveal a rich network of connectivity between neighboring layer 6 neurons (Briggs and Callaway, 2001; Binzegger et al., 2004). In considering the circuits that contribute to the wide-field response, it should be pointed out that this response could reflect direct excitation and/or release from inhibition.

Finally, the early and transient wide-field excitation described here could provide an important utility for visual processing. Wide-field excitation could provide postsynaptic neurons with information about the global context in which the receptive field is embedded including global contrast and feature orientation. Fast and transient wide-field excitation could also signal the presence of salient features within a larger visual context that requires attention. With these possibilities in mind, it would be interesting to determine whether the inputs establishing fast and transient wide-field excitation use burst spiking, or other rapid and transient modes of communication and whether these sources of input are influenced by transitions between nonattentive and attentive states (Llinás and Steriade, 2006).

\section{References}

Adelson E, Bergen J (1985) Spatiotemporal energy models for the perception of motion. J Opt Soc Am 2:284-299.

Alitto HJ, Usrey WM (2008) Origin and dynamics of extraclassical suppression in the lateral geniculate nucleus of the macaque monkey. Neuron 57:135-146.

Angelucci A, Bressloff PC (2006) Contribution of feedforward, lateral and feedback connections to the classical receptive field center and extraclassical receptive field surround of primate V1 neurons. Prog Brain Res 154:93-120.

Angelucci A, Levitt JB, Walton EJS, Hupe J-M, Bullier J, Lund JS (2002) Circuits for local and global signal integration in primary visual cortex. J Neurosci 22:8633-8646.

Bair W, Cavanaugh JR, Movshon JA (2003) Time course and time-distance relationships for surround suppression in macaque V1 neurons. J Neurosci 23:7690-7701.

Binzegger T, Douglas RJ, Martin KAC (2004) A quantitative map of the circuit of cat primary visual cortex. J Neurosci 24:8441-8453.

Bonin V, Mante V, Carandini M (2005) The suppressive field of neurons in lateral geniculate nucleus. J Neurosci 25:10844-10856.

Briggs F, Callaway EM (2001) Layer-specific input to distinct cell types in layer 6 of monkey primary visual cortex. J Neurosci 21:3600-3608.

Briggs F, Usrey WM (2007) A fast, reciprocal pathway between the lateral geniculate nucleus and visual cortex in the macaque monkey. J Neurosci 27:5431-5436.

Briggs F, Usrey WM (2009) Parallel processing in the corticogeniculate pathway of the macaque monkey. Neuron 62:135-146.

Cai Y, Zhou T, Chen L (2008) Effects of binocular suppression on surround suppression. J Vis 18:9.1-10.

Callaway EM (2004) Cell types and local circuits in primary visual cortex of the macaque monkey. In: The visual neurosciences, Vol 1 (Chalupa L, Werner J, eds), pp 680-694. Cambridge, MA: MIT Press.

Camp AJ, Tailby C, Solomon SG (2009) Adaptable mechanisms that regulate the contrast response of neurons in the primate lateral geniculate nucleus. J Neurosci 29:5009-5021.

Carandini M, Heeger DJ, Senn W (2002) A synaptic explanation of suppression in visual cortex. J Neurosci 22:10053-10065.

Cavanaugh JR, Bair W, Movshon JA (2002a) Nature and interaction of signals from the receptive field center and surround in macaque V1 neurons. J Neurophysiol 88:2530-2546.

Cavanaugh JR, Bair W, Movshon JA (2002b) Selectivity and spatial distri- bution of signals from the receptive field surround in macaque V1 neurons. J Neurophysiol 88:2547-2556.

Chen X, Han F, Poo MM, Dan Y (2007) Excitatory and suppressive receptive field subunits in awake monkey primary visual cortex (V1). Proc Natl Acad Sci U S A 104:19120-19125.

DeAngelis GC, Ohzawa I, Freeman RD (1995) Receptive-field dynamics in the central visual pathways. Trends Neurosci 18:451-458.

Durand S, Freeman TCB, Carandini M (2007) Temporal properties of surround suppression in cat primary visual cortex. Vis Neurosci 24:679-690.

Felleman DJ, Van Essen DC (1991) Distributed hierarchical processing in the primate cerebral cortex. Cereb Cortex 1:1-47.

Fitzpatrick D, Usrey WM, Schofield BR, Einstein G (1994) The sublaminar organization of corticogeniculate neurons in layer 6 of macaque striate cortex. Vis Neurosci 11:307-315.

Gilbert CD (1977) Laminar differences in receptive field properties of cells in cat primary visual cortex. J Physiol 268:391-421.

Grieve KL, Sillito AM (1995) Differential properties of cells in the feline primary visual cortex providing the corticofugal feedback to the lateral geniculate nucleus and visual claustrum. J Neurosci 15:4868-4874.

Ichida JM, Schwabe L, Bressloff PC, Angelucci A (2007) Response facilitation from the "suppressive" receptive field surround in macaque V1 neurons. J Neurophysiol 98:2168-2181.

Jones HE, Grieve KL, Wang W, Sillito AM (2001) Surround suppression in primate V1. J Neurophysiol 86:2011-2028.

Jones HE, Wang W, Sillito AM (2002) Spatial organization and magnitude of orientation contrast interactions in primate V1. J Neurophysiol 88:2796-2808.

Kapadia MK, Westheimer G, Gilbert CD (1999) Dynamics of spatial summation in primary visual cortex of alert monkeys. Proc Natl Acad Sci U S A 96:12073-12078.

Knierim JJ, van Essen DC (1992) Neuronal responses to static texture patterns in area V1 of the alert macaque monkey. J Neurophysiol 67:961-980.

Levitt JB, Lund JS (2002) The spatial extent over which neurons in macaque striate cortex pool visual signals. Vis Neurosci 19:439-452.

Liu Y-J, Hashemi-Nezhad M, Lyon DC (2011) Dynamics of extraclassical surround modulation in three types of V1 neurons. J Neurophysiol 105:1306-1317.

Llinás RR, Steriade M (2006) Bursting of thalamic neurons and states of vigilance. J Neurophysiol 95:3297-3308.

Lund JS, Lund RD, Hendrickson AE, Bunt AH, Fuchs AF (1975) The origin of efferent pathways from the primary visual cortex, area 17, of the macaque monkey as shown by retrograde transport of horseradish peroxidase. J Comp Neurol 164:287-303.

Mareschal I, Shapley RM (2004) Effects of contrast and size on orientation discrimination. Vis Res 44:57-67.

Naito T, Sadakane O, Okamoto M, Sato H (2007) Orientation tuning of surround suppression in lateral geniculate nucleus and primary visual cortex of cat. Neuroscience 149:962-975.

Nolt MJ, Kumbhani RD, Palmer LA (2004) Contrast-dependent spatial summation in the lateral geniculate nucleus and retina of the cat. J Neurophysiol 92:1708-1717.

Nothdurft HC, Gallant JL, Van Essen DC (1999) Response modulation by texture surround in primate area V1: correlates of "popout" under anesthesia. Vis Neurosci 16:15-34.

Ozeki H, Sadakane O, Akasaki T, Naito T, Shimegi S, Sato H (2004) Relationship between excitation and inhibition underlying size tuning and contextual response modulation in the cat primary visual cortex. J Neurosci 24:1428-1438.

Rust NC, Schwartz O, Movshon JA, Simoncelli EP (2005) Spatiotemporal elements of macaque V1 receptive fields. Neuron 46:945-956.

Sadakane O, Ozeki H, Naito T, Akasaki T, Kasamatsu T, Sato H (2006) Contrast-dependent, contextual response modulation in primary visual cortex and lateral geniculate nucleus of the cat. Eur J Neurosci 23:1633-1642.

Sceniak MP, Ringach DL, Hawken MJ, Shapley R (1999) Contrast's effect on spatial summation by macaque V1 neurons. Nat Neurosci 2:733-739.

Sceniak MP, Hawken MJ, Shapley R (2001) Visual spatial characterization of macaque V1 neurons. J Neurophysiol 85:1873-1887.

Sceniak MP, Chatterjee S, Callaway EM (2006) Visual spatial summa- 
tion in macaque geniculocortical afferents. J Neurophysiol 96:34743484.

Schwabe L, Ichida JM, Shushruth S, Mangapathy P, Angelucci A (2010) Contrast-dependence of surround suppression in macaque V1: experimental testing of a recurrent network model. Neuroimage 52: 777-792.

Sincich LC, Horton JC (2005) The circuitry of V1 and V2: integration of color, form, and motion. Annu Rev Neurosci 28:303-326.

Smith MA, Bair W, Movshon JA (2006) Dynamics of suppression in macaque primary visual cortex. J Neurosci 26:4826-4834.

Solomon SG, Lee BB, Sun H (2006) Suppressive surrounds and contrast gain in magnocellular-pathway retinal ganglion cells of macaque. J Neurosci 26:8715-8726.

Sullivan TJ, de Sa VR (2006) A model of surround suppression through cortical feedback. Neural Netw 19:564-572.
Tailby C, Solomon SG, Peirce JW, Metha AB (2007) Two expressions of "surround suppression" in V1 that arise independent of cortical mechanisms of suppression. Vis Neurosci 24:99-109.

Valberg A, Lee BB, Tigwell DA, Creutzfeldt OD (1985) A simultaneous contrast effect of steady remote surrounds on responses of cells in macaque lateral geniculate nucleus. Exp Brain Res 58:604-608.

Wang C, Bardy C, Huang JY, FitzGibbon T, Dreher B (2009) Contrast dependence of center and surround integration in primary visual cortex of the cat. J Vis 9:20.1-15.

Webb BS, Tinsley CJ, Barraclough NE, Parker A, Derrington AM (2003) Gain control from beyond the classical receptive field in primate visual cortex. Vis Neurosci 20:221-230.

Webb BS, Dhruv NT, Solomon SG, Tailby C, Lennie P (2005) Early and late mechanisms of surround suppression in striate cortex of macaque. J Neurosci 25:11666-11675. 